\title{
The prevalence and reliability of self-reported penicillin allergy in a community hospital
}

This article was published in the following Dove Press journal:

International Journal of General Medicine

5 December 2013

Number of times this article has been viewed

\section{Faisal A Khasawneh' \\ Megan A R Slaton ${ }^{2}$ \\ Stephen L Katzen ${ }^{2}$ \\ Ashley A Woolbert ${ }^{2}$ \\ Sean D Anderson ${ }^{2}$ \\ Michelle B Parker ${ }^{2}$ \\ Rachel MAnderson ${ }^{2}$ \\ Krystal K Haase ${ }^{3}$ \\ Roger D Smalligan ${ }^{4}$}

'Section of Infectious Diseases, Department of Internal Medicine, ${ }^{2}$ School of Medicine, ${ }^{3}$ School of Pharmacy, ${ }^{4}$ Department of Internal Medicine, Texas Tech University Health Sciences Center, Amarillo, TX, USA
Correspondence: Faisal A Khasawneh Section of Infectious Diseases,

Department of Internal of Medicine, Texas Tech University Health Sciences Center, 1400 S Coulter Street,

Amarillo, TX 79106, USA

Tel +l 8063545480

Fax + I 8063545765

Email faisal.khasawneh@ttuhsc.edu
Background: Penicillin (PCN) accounts for most cases of antibiotic allergies. Reported PCN allergy deprives the patient from this class of antibiotics and creates hesitancy in using other beta-lactam antibiotics. The aim of this study is to report the prevalence of self-reported PCN allergy among adult patients admitted to the hospital and to examine the probable validity of these reports.

Methods: A questionnaire was conducted among 192 patients with self-reported PCN allergy who were admitted to a community hospital between July 25, 2011 and January 25, 2012. Patients admitted with an infection and treated with a beta-lactam were also followed until hospital discharge.

Results: The mean age of patients at the time of their self-reported allergic reaction was 20.3 years. The most common allergic symptoms reported in decreasing order of frequency were itchy rash, angioedema, and urticaria. Based on analysis of the questionnaires, 121 patients (63.0\%) had probable PCN allergy, 54 (28.1\%) had possible PCN allergy, and 17 (8.9\%) were unlikely to have a PCN allergy. Fifty-one participants (26.6\%) had self-reported subsequent exposure to PCN in their life. This subsequent exposure was well tolerated in $86.3 \%$ of the participants. Fifty participants $(25.9 \%)$ had self-reported subsequent exposure to a first generation cephalosporin and it was well tolerated in $78.4 \%$ of them.

Conclusion: Taking a detailed history from patients with self-reported PCN allergy can help to distinguish a true PCN allergy from a false positive report of allergy and hence allow clinicians to use this important class of antibiotics when truly indicated.

Keywords: penicillins, beta-lactam antibiotics, allergy

\section{Introduction}

Antibiotic allergy accounts for a small but significant proportion of adverse drug reactions. ${ }^{1,2}$ These allergic reactions are immunologically mediated and can affect multiple organ systems with variable degrees of severity. ${ }^{3}$ The most common allergic reactions to antibiotics are rashes, including urticaria, and pruritus. ${ }^{4,5}$ After first exposure to an antibiotic there is typically a period of sensitization ranging from days to weeks before an adverse reaction is noted. ${ }^{6}$ On subsequent exposure to the same antibiotic in a previously sensitized person the allergic reaction can appear within minutes to hours.

The majority of antibiotic allergies are self-reported. ${ }^{7}$ The most common antibiotic patients report as an allergy is penicillin $(\mathrm{PCN}){ }^{7}$ Penicillins and other beta-lactam antibiotics are the cornerstone of empiric antibiotic regimens for life threatening infections and are the preferred treatment for many infections once culture and sensitivity results 
are identified. ${ }^{8-10}$ Reported allergy to PCN, if inaccurate, deprives patients of this important class of antibiotics and makes clinicians hesitant to use other beta-lactam antibiotics out of fear from cross-allergy.

This study was designed to measure the prevalence and probable accuracy or reliability of reported PCN allergy among adult inpatients admitted to a busy teaching internal medicine service in West Texas. Furthermore, the reliability of the self-reported PCN allergy was tested by obtaining detailed history supporting true allergic reaction and by examining the medical records for documented reactions after beta-lactam antibiotics exposure during the patients' current hospitalization.

\section{Methods}

This was a prospective observational study conducted at Northwest Texas Hospital (NWTH) in Amarillo, Texas on the Tech University Health Sciences Center (TTUHSC) internal medicine service. NWTH is a 400 bed community teaching hospital in Amarillo, Texas. The study was approved by the Institutional Review Boards for both TTUHSC and NWTH. Between July 25, 2011 and January 25, 2012 the investigators reviewed the medical records of all newly admitted TTUHSC patients on a daily basis, specifically looking for documentation of a reported PCN allergy in the nursing, pharmacy, and physician sections. Patients with allergy to beta-lactams other than PCN were not included. Patients with an alleged PCN allergy were invited by the investigators to participate in the study and were asked to sign an informed consent document. In the event of the patient being unable to answer questions (due to intubation, critical illness, or delirium), their next of kin were approached and asked to participate. Subjects were interviewed using a standardized questionnaire (Table 1).

The patient's age, sex, other antibiotic allergies, and reason for admission were collected from medical records.

Table I Standardized questionnaire used in the study

I. What was the patient's age at the time of the reaction?

2. Does the patient recall the reaction? If not, who informed him of it?

3. How long after starting the PCN course did the reaction begin?

4. What were the characteristics of the reaction?

5. What was the route of antibiotic administration?

6. Why was PCN prescribed for the patient?

7. Has the patient taken any of the following beta-lactam antibiotics: amoxicillin, amoxicillin/clavulanate, or cephalexin after his/her initial $\mathrm{PCN}$ allergic reaction? If yes, did he/she have a reaction to it?

Abbreviation: $\mathrm{PCN}$, penicillin.
Patients admitted with an infection and who were being treated with a beta-lactam antibiotic were followed prospectively throughout their admission for any sign of allergic reaction attributed to that antibiotic.

Based on the participants' answers to the questionnaire, patients were divided into one of three categories: 1) probable PCN allergy; 2) possible PCN allergy; or 3) unlikely to have PCN allergy. Criteria for placement in each of the categories above were as follows: 1) probable PCN allergy: if the reaction was a) documented in the medical record of a previous admission to NWTH, b) if the same type of reaction had occurred on two different occasions after exposure to PCN, or c) if the patient's reported signs and symptoms were consistent with an allergic reaction (itchy rash, urticaria, angioedema, bronchospasm, or anaphylactic shock); 2) possible PCN allergy: if the patient reported a) a nonitchy rash only, b) typical signs/symptoms (itchy rash, urticaria, angioedema, bronchospasm, or anaphylactic shock) with uneventful subsequent exposure to $\mathrm{PCN}$, or c) if the patient could not recall the signs or symptoms that caused him/her to be labeled PCN allergic; and 3) unlikely to have PCN allergy: if the patient reported signs or symptoms consistent with a known side effect of PCN and was mistakenly labeled as allergic.

\section{Statistical analysis}

For continuous variables, the mean with standard deviation was reported. For categorical variables, the number of patients with that variable and a corresponding percentage were reported. Univariate comparisons between the above three patient categories were performed by ordered logistic regression (robust). A $P$-value of less than 0.05 was considered statistically significant. The statistical analysis was done using Stata 12 (StataCorp LP, College Station, TX, USA).

\section{Results}

During the study period, 2,589 patients were admitted to NWTH on the TTUHSC internal medicine service. Self-reported PCN allergy was found to be documented in the charts of $387(14.9 \%)$ patients. After being asked to participate in the study, 192 patients $(49.6 \%)$ consented and answered the questionnaire, 89 patients $(23.0 \%)$ declined, 3 patients $(0.8 \%)$ did not speak English, 26 patients $(6.7 \%)$ had mental status changes and no next of kin was available to consent, 19 patients (4.9\%) died before being approached by the investigators, and 58 patients (15.0\%) were discharged before being approached to participate. The patient provided the information in 127 of the encounters $(66.1 \%)$, 
while next of kin (spouse in 15 cases, parents in 11 cases, and a sibling in 7 cases) was the source of information in the rest. After applying the above criteria to the provided answers in the questionnaires, 121 patients $(63.0 \%)$ were classified as probable $\mathrm{PCN}$ allergy, 54 patients $(28.1 \%)$ as possible PCN allergy, and 17 patients $(8.9 \%)$ as unlikely to have PCN allergy. The characteristics of each group are shown in Table 2.

The mean age of patients at the time of allergic reaction was $20.3 \pm 15.0$ years. The most common reasons for having been prescribed PCN were pharyngitis and other respiratory tract infections, which accounted for $44 \%$ of cases. The most common allergic symptoms reported were itchy rash followed by angioedema and urticaria. Reported side effects mislabeled as allergic reactions were abdominal pain, heart burn, nausea, vomiting, hair loss, confusion, and seizures.

Thirty-six patients reported allergies to other antibiotics in the following order: sulfa, cephalosporins, erythromycin, fluoroquinolones, tetracycline, vancomycin, and metronidazole. Forty-nine patients (26.6\%) with alleged PCN allergy reported subsequent exposure to amoxicillin or amoxicillin/clavulanate, including seven patients with probable PCN allergy, and 42 of them $(85.7 \%)$ tolerated it well. None of the above mentioned seven patients with probable PCN allergy tolerated subsequent
PCN exposure. Fifty-one patients (26.6\%) with alleged PCN allergy reported subsequent exposure to cephalexin, including 19 patients with probable PCN allergy, and 40 of them (78.4\%) tolerated it well.

Forty-two patients $(21.9 \%)$ were admitted to the hospital with an infection. All of the 24 patients who received a broad spectrum (third generation and above) cephalosporin (16 patients) or a carbapenem (8 patients) tolerated it well. One patient with probable PCN allergy received aztreonam and, expectedly, tolerated it well.

\section{Discussion}

Previous reports in the medical literature have estimated that $10 \%-20 \%$ of patients with a self-reported PCN allergy are ultimately found to be truly allergic when assessed by skin testing. ${ }^{11}$ The reasons behind this over reporting of PCN allergy include patient confusion of side effects for allergic reactions, being informed of their alleged allergy by a parent without a firsthand recall of the reaction, and loss of the specific immunoglobulin E (IgE) conferring PCN allergy. ${ }^{12,13}$ As demonstrated, taking a detailed history in these cases may allow clinicians to exclude true PCN allergy, permitting patients to receive the antibiotic in question without serious sequelae. Patients with a concerning history for a serious

Table 2 The characteristics of 192 patients with reported penicillin allergy

\begin{tabular}{|c|c|c|c|c|}
\hline Characteristic & $\begin{array}{l}\text { Probable } \\
\text { PCN allergy }\end{array}$ & $\begin{array}{l}\text { Possible } \\
\text { PCN allergy }\end{array}$ & $\begin{array}{l}\text { Unlikely } \\
\text { PCN allergy }\end{array}$ & $\begin{array}{l}\text { Groups difference (ordered logistic } \\
\text { regression [robust]) }\end{array}$ \\
\hline Number of patients & 121 & 54 & 17 & \\
\hline Age (years $\pm S D)$ & $55.1 \pm 17.0$ & $55.6 \pm 17.2$ & $55.4 \pm 13.6$ & $P=0.874$ \\
\hline Sex & & & & $P=0.136$ \\
\hline Male & 50 & 25 & 11 & \\
\hline Female & 71 & 29 & 6 & \\
\hline Onset of allergic reaction* & & & & $P=0.112$ \\
\hline Childhood ( $\leq 10$ yrs) & 42 & 27 & 6 & \\
\hline $11-19$ yrs & 25 & 8 & 0 & \\
\hline Adulthood ( $\geq 20$ yrs) & 53 & 18 & 11 & \\
\hline Route of exposure** & & & & $P=0.643$ \\
\hline Intramuscular inj & 62 & 25 & 5 & \\
\hline Intravenous inj & 13 & 8 & 1 & \\
\hline Oral & 40 & 14 & 9 & \\
\hline Time from exposure to allergic reaction $* * *$ & & & & $P=0.012$ \\
\hline Within 24 hours & 76 & 22 & 7 & \\
\hline After 24 hours & 24 & 14 & 8 & \\
\hline Tolerated PCN on subsequent exposure & 0 out of 7 & 37 out of 37 & 5 out of 5 & NA \\
\hline Tolerated cephalexin on subsequent exposure & 19 out of 28 & 18 out of 20 & 3 out of 3 & $P=0.049$ \\
\hline Admitted with an infection & 25 & 11 & 6 & $P=0.477$ \\
\hline Received a beta-lactam & 14 & 6 & 5 & $P=0.284$ \\
\hline Other antibiotic allergies & 24 & 9 & 3 & $P=0.637$ \\
\hline
\end{tabular}

Notes: *Denotes that two patients could not remember the age at which they had the penicillin allergic reaction; **denotes that 15 patients could not remember the route of penicillin exposure; ***denotes that $4 \mathrm{I}$ patients could not remember the time from penicillin exposure to allergic reaction. Abbreviations: inj, injection; NA, not applicable; PCN, penicillin; SD, standard deviation. 


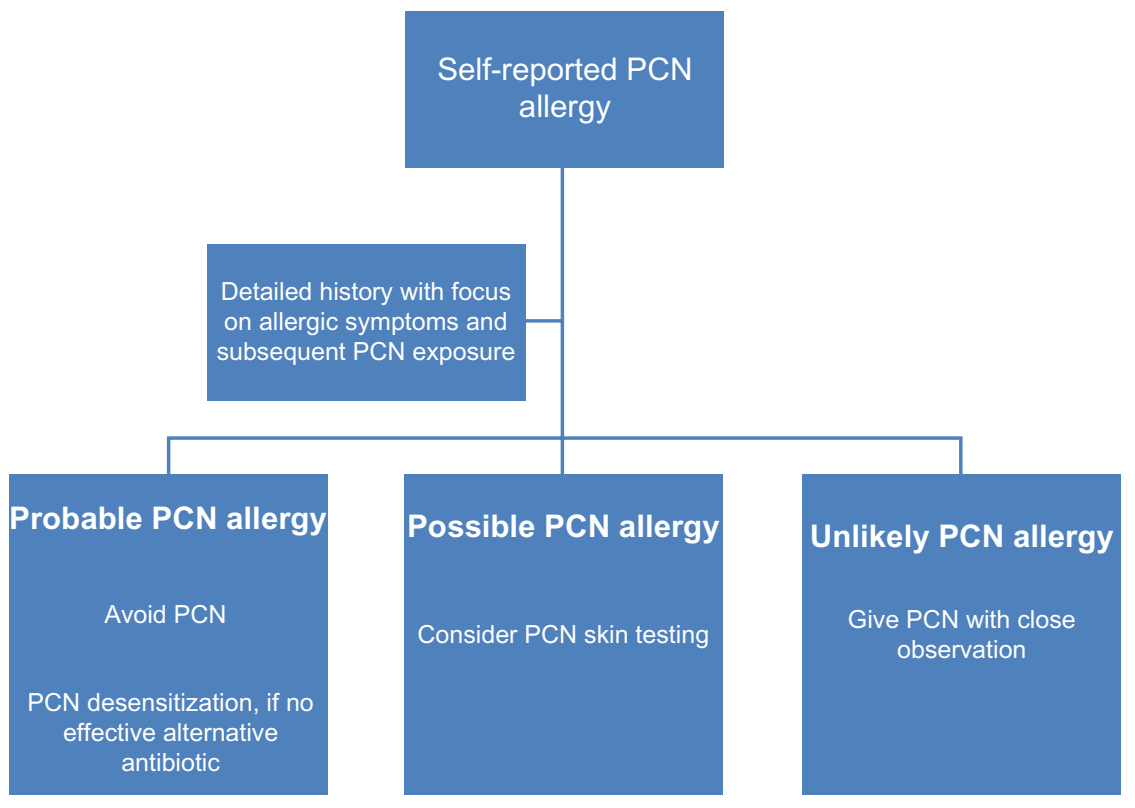

Figure I Algorithm incorporating the current study findings in clinical practice. Abbreviation: PCN, penicillin.

allergic reaction and a compelling need for that antibiotic should undergo skin testing and subsequent PCN desensitization if indicated. ${ }^{3,14}$ Figure 1 demonstrates how we have incorporated the findings of this study into our practice.

It has been demonstrated that the majority of IgEmediated reactions occur within 1 hour of PCN exposure and that they rarely develop after 72 hours. ${ }^{6}$ Hence, one of the questions used to help determine if a reaction was likely to be related to PCN exposure was the time from exposure to developing the reaction. In our population, patients ultimately deemed to have either a probable or possible PCN allergy were more likely to display signs and symptoms consistent with allergy within 24 hours of exposure, as compared to patients felt unlikely to have PCN allergy ( $P=0.012)$.

An often mentioned concern of clinicians when discussing PCN allergy is the possibility of cross-allergy with cephalosporins. ${ }^{15,16} \mathrm{PCN}$ and cephalosporins have similar beta-lactam rings but the beta-lactam rings of cephalosporins undergo a more rapid fragmentation in vivo than those of PCNs making immunologic cross-reactivity uncommon. ${ }^{17}$ Several studies have suggested that PCN and cephalosporin cross-reactivity depends on sharing similar side chain substitutes rather than similar beta-lactam rings. ${ }^{18}$ Higher PCNcephalosporin cross-allergy rates previously reported have been attributed to PCN test compounds being contaminated with cephalosporins. ${ }^{19}$ Patients with true PCN allergy have a 3-fold increase in adverse antibiotic reactions in general, irrespective of any noted structural similarity. ${ }^{6}$
Cephalosporins themselves cause allergic reactions among $1 \%-3 \%$ of patients, whether the patient is allergic to PCN or not. ${ }^{19}$ Currently, cross-allergy between $\mathrm{PCN}$ and cephalosporins is uncommon, occurring in less than $5 \%$ of cases, with the majority of these reactions being found between PCN and first generation cephalosporins rather than between PCN and advanced generation cephalosporins. ${ }^{15}$ This fact held true in our study where patients with probable PCN allergy were more likely to have a reaction to cephalexin (first generation cephalosporin) on subsequent exposure compared to patients with possible or unlikely PCN allergy $(P=0.049)$. In addition, there was no cross-allergy between PCN and the third and fourth generation cephalosporins seen in our cohort of patients.

Carbapenems were well tolerated in our study group. Romano et al tested meropenem tolerance among patients with IgE-mediated hypersensitivity to PCN and found that among 104 patients with a positive PCN skin test only one patient had a positive skin test to meropenem (cross-allergy prevalence of $0.9 \%) .{ }^{20}$ All patients with a negative meropenem skin test also had no reaction to a subsequent intravenous meropenem challenge. In a similar study of imipenem involving 112 patients with immediate hypersensitivity to PCN, only one patient $(0.9 \%)$ had a positive skin test and the remainder tolerated an intramuscular imipenem challenge without any clinical reaction. ${ }^{21}$

The current study has several limitations. First, recall bias was a factor since patients may or may not have remembered allergic symptoms and subsequent antibiotic exposures 
accurately. This problem was compounded when the allergy history had to be obtained from a family member due to the patient being ventilated or incapacitated in some way. The second limitation was the lack of PCN skin testing to provide further confirmation or clarification of the designated patient groups. At the time of the study, the now commercially available product Pre-pen ${ }^{\circledR}$ (ALK, Round Rock, TX, USA) which can be used for PCN skin testing at the bedside was not available due to a national shortage. Thirdly, the focus of the study was limited to life threatening IgE-mediated "type I" allergic reactions. Symptoms and signs suggesting antibody mediated, immune complex mediated and delayed hypersensitivity reactions were not sought.

In conclusion, taking a detailed history from patients with self-reported PCN allergy by focusing on allergic symptoms and subsequent PCN exposure can help exclude true allergy and allow the use of this important class of antibiotics when clinically indicated. Furthermore, this study supports the concept that patients with self-reported PCN allergy generally tolerate other beta-lactam antibiotics well, with minimal risk of cross-allergy.

\section{Disclosure}

All authors report no conflicts of interest in this work.

\section{References}

1. Lazarou J, Pomeranz BH, Corey PN. Incidence of adverse drug reactions in hospitalized patients: a meta-analysis of prospective studies. JAMA. 1998;279(15):1200-1205.

2. Pirmohamed M, James S, Meakin S, et al. Adverse drug reactions as cause of admission to hospital: prospective analysis of 18820 patients. BMJ. 2004;329(7456):15-19.

3. Arroliga ME, Pien L. Penicillin allergy: consider trying penicillin again. Cleve Clin J Med. 2003;70(4):313-314, 307-308, 320-321 passim.

4. Fiszenson-Albala F, Auzerie V, Mahe E, et al. A 6-month prospective survey of cutaneous drug reactions in a hospital setting. Br J Dermatol. 2003;149(5):1018-1022.

5. Lee CE, Zembower TR, Fotis MA, et al. The incidence of antimicrobial allergies in hospitalized patients: implications regarding prescribing patterns and emerging bacterial resistance. Arch Intern Med. 2000; 160(18):2819-2822.

6. Salkind AR, Cuddy PG, Foxworth JW. The rational clinical examination. Is this patient allergic to penicillin? An evidence-based analysis of the likelihood of penicillin allergy. JAMA. 2001;285(19):2498-2505.

7. Gruchalla RS, Pirmohamed M. Clinical practice. Antibiotic allergy. N Engl J Med. 2006;354(6):601-609.
8. Tunkel AR, Hartman BJ, Kaplan SL, et al. Practice guidelines for the management of bacterial meningitis. Clin Infect Dis. 2004;39(9): 1267-1284.

9. Mandell LA, Wunderink RG, Anzueto A, et al; Infectious Diseases Socieity of America; American Thoracic Society. Infectious Diseases Society of America/American Thoracic Society consensus guidelines on the management of community-acquired pneumonia in adults. Clin Infect Dis. 2007;44 Suppl 2:S27-S72.

10. Baddour LM, Wilson WR, Bayer AS, et al; Committee on Rheumatic Fever, Endocarditis, and Kawasaki Disease; Council on Cardiovascular Disease in the Young; Councils on Clinical Cardiology, Stroke, and Cardiovascular Surgery and Anesthesia; Americam Heart Association; Infectious Diseases Society of America. Infective endocarditis: diagnosis, antimicrobial therapy, and management of complications: a statement for healthcare professionals from the Committee on Rheumatic Fever, Endocarditis, and Kawasaki Disease, Council on Cardiovascular Disease in the Young, and the Councils on Clinical Cardiology, Stroke, and Cardiovascular Surgery and Anesthesia, American Heart Association: endorsed by the Infectious Diseases Society of America. Circulation. 2005;111(23):e394-e434.

11. Sogn DD, Evans R 3rd, Shepherd GM, et al. Results of the National Institute of Allergy and Infectious Diseases Collaborative Clinical Trial to test the predictive value of skin testing with major and minor penicillin derivatives in hospitalized adults. Arch Intern Med. 1992;152(5): 1025-1032.

12. Robinson JL, Hameed T, Carr S. Practical aspects of choosing an antibiotic for patients with a reported allergy to an antibiotic. Clin Infect Dis. 2002;35(1):26-31.

13. Sullivan TJ, Wedner HJ, Shatz GS, Yecies LD, Parker CW. Skin testing to detect penicillin allergy. J Allergy Clin Immunol. 1981; 68(3):171-180.

14. Forrest DM, Schellenberg RR, Thien VV, King S, Anis AH, Dodek PM. Introduction of a practice guideline for penicillin skin testing improves the appropriateness of antibiotic therapy. Clin Infect Dis. 2001;32(12): 1685-1690.

15. Pichichero ME. A review of evidence supporting the American Academy of Pediatrics recommendation for prescribing cephalosporin antibiotics for penicillin-allergic patients. Pediatrics. 2005;115(4):1048-1057.

16. Campagna JD, Bond MC, Schabelman E, Hayes BD. The use of cephalosporins in penicillin-allergic patients: a literature review. J Emerg Med. 2012;42(5):612-620.

17. Pichichero ME, Casey JR. Safe use of selected cephalosporins in penicillin-allergic patients: a meta-analysis. Otolaryngol Head Neck Surg. 2007;136(3):340-347.

18. Pichichero ME. Use of selected cephalosporins in penicillinallergic patients: a paradigm shift. Diagn Microbiol Infect Dis. 2007; 57(Suppl 3):13S-18S.

19. Kelkar PS, Li JT. Cephalosporin allergy. $N$ Engl J Med. 2001; 345(11):804-809.

20. Romano A, Viola M, Gueant-Rodriguez RM, Gaeta F, Valluzzi R, Gueant JL. Brief communication: tolerability of meropenem in patients with IgE-mediated hypersensitivity to penicillins. Ann Intern Med. 2007;146(4):266-269.

21. Romano A, Viola M, Gueant-Rodriguez RM, Gaeta F, Pettinato R, Gueant JL. Imipenem in patients with immediate hypersensitivity to penicillins. N Engl J Med. 2006;354(26):2835-2837.
International Journal of General Medicine

\section{Publish your work in this journal}

The International Journal of General Medicine is an international, peer-reviewed open-access journal that focuses on general and internal medicine, pathogenesis, epidemiology, diagnosis, monitoring and treatment protocols. The journal is characterized by the rapid reporting of reviews, original research and clinical studies across all disease areas.

\section{Dovepress}

A key focus is the elucidation of disease processes and management protocols resulting in improved outcomes for the patient. The manuscript management system is completely online and includes a very quick and fair peer-review system. Visit http://www.dovepress.com/ testimonials.php to read real quotes from published authors. 